\title{
DNA methylation in normal and malignant hematopoiesis
}

\author{
Hamza Celik ${ }^{1} \cdot{\text { Ashley } \text { Kramer }^{1} \cdot \text { Grant A. Challen }}^{1,2}$
}

Received: 28 January 2016 / Accepted: 8 February 2016 / Published online: 4 March 2016

(C) The Japanese Society of Hematology 2016

\begin{abstract}
The study of DNA methylation has been a rapidly expanding field since its dawn in the 1960s. DNA methylation is an epigenetic modification that plays a crucial role in guiding the differentiation of stem cells to their destined lineage, and in maintaining tissue homeostasis. Moreover, aberrant DNA methylation has been well characterized as a significant contributing factor in the pathogenesis of a variety of cancers. Hematopoiesis is a process that is uniquely susceptible to epigenetic changes due to the small pool of actively cycling stem cells that give rise to the entire mature immune-hematopoietic system. Mutations in DNA methyltransferase enzymes have been shown to be initiating events in the development of hematological malignancies such as acute myeloid leukemia and, therefore, have become targets for improved diagnostics and therapy. The spatial and temporal regulation of DNA methylation in the hematopoietic developmental hierarchy is critical to hematopoietic homeostasis. An improved understanding of the roles that DNA methylation plays in normal and malignant hematopoiesis will have a significant impact on the future of regenerative stem cell therapy and clinical treatment of hematopoietic malignancies. This review aims to highlight current developments in the field and prioritize future research directions.
\end{abstract}

Grant A. Challen

gchallen@dom.wustl.edu

1 Section of Stem Cell Biology, Division of Oncology, Department of Internal Medicine, Washington University in St. Louis School of Medicine, 660 Euclid Avenue, St. Louis, MO 63110, USA

2 Developmental, Regenerative and Stem Cell Biology Program, Division of Biology and Biomedical Sciences, Washington University in St. Louis School of Medicine, St. Louis, MO 63110, USA
Keywords DNA methylation · Hematopoiesis · Leukemic transformation

\section{Introduction}

Hematopoietic stem cells (HSCs) that reside in the bone marrow (BM) ensure lifelong blood and BM homeostasis by giving rise to all cells of the hematopoietic lineages, while maintaining self-renewal capacity. Hematopoiesis proceeds in a hierarchical manner, giving rise to progenitors with limited self-renewal ability that differentiate into lineage-restricted cells, eventually giving rise to all the various cells of the immune-hematopoietic system. The balance between HSC self-renewal and differentiation is tightly controlled by number of mechanisms that include transcription factors, signal transduction pathways, and niche factors (Reviews 1-4). In addition, recent studies provided overwhelming evidence suggesting that epigenetic factors, such as DNA methylation, act in concert with these transcriptional factors to ensure hematopoietic homeostasis. Improper orchestration of epigenetic mechanisms that control this balance can cause aberrant HSC function and induce a number of hematologic disorders/malignancies, such as leukemias and lymphomas, myeloproliferative neoplasms (MPN), myelodysplastic syndromes (MDS), and multiple myeloma (MM) (Reviews 1-4).

In the mammalian genome, DNA methylation of $\mathrm{CpG}$ dinucleotides is one of the most common forms of epigenetic modifications, and is essential for normal development, influencing processes such as, X-chromosome inactivation, genomic imprinting, and chromosome structure and stability $[5,6]$. DNA methylation is catalyzed at $\mathrm{CpG}$ sites by at least three different DNA methyltransferases (DNMTs) [7]: DNMT1, DNMT3A, and DNMT3B, by 
transferring a methyl group to the fifth carbon of a cytosine residue to form 5-methylcytosine. DNMT3A and DNMT3B are known as de novo DNMTs and can establish new methylation patterns to unmodified DNA [8, 9]. DNMT1 acts as a maintenance methyltransferase by recognizing hemimethylated DNA and copying the pre-existing DNA methylation pattern from the parental DNA strand onto the newly synthesized daughter strand during replication [10].

The importance of proper orchestration of DNA methylation throughout mammalian development is made evident through mouse models of germline gene deletion which show that constitutive loss of Dnmt1 causes developmental termination at gastrulation [11]. Dnmt3b-null embryos exhibit growth impairment and neural tube defects causing termination of development at E9.5, and Dnmt3a-null mice appear normal at birth but shortly thereafter growth is severely stunted and mice die at approximately 4 weeks of age [9]. Germline Dnmt3a/3b double knockout embryos terminate shortly after gastrulation as indicated by a lack of somites in mutant embryos [9]. To date, the precise mechanism of how DNA methylation patterns influence gene expression and how they vary between cell types is still poorly understood. However, transcriptional repression via DNA methylation is thought to occur through a number of mechanisms such as direct inhibition of transcriptional activators from their target promoters, the recruitment of co-repressor complexes by methyl CpG-binding proteins that recognize methylated regions of DNA, and co-operation with histone modification proteins leading to structural changes in the chromatin. In this review, we explore current knowledge of the innate functions of the aforementioned DNA methyltransferases in hematopoiesis and the consequences of mutations in these proteins that lead to hematological malignancies.

\section{Role of DNMTs in normal hematopoiesis}

\section{DNMT1}

The maintenance Dnmt, Dnmt1, has been shown to be essential for HSC self-renewal, niche retention and multilineage hematopoietic differentiation [12, 13]. The conditional knockout (KO) of Dnmt1 in the hematopoietic system using the Mx1-Cre driver leads to severe genomic hypomethylation in HSCs, which is followed by hematopoietic pancytopenia and a rapid death within 10 days post-induction by injection of polyinosinic:polycytidylic acid (pIpC). Deletion of Dnmt1 in hematopoiesis eliminates HSCs and bone marrow progenitors by the induction of cell-autonomous apoptosis [12]. There is, however, a controversy in the aforementioned effect of conditional knockout of Dnmt1 in HSCs of primary mice due to the finding by Trowbridge et al. in the same year that Mx1Cre:Dnmt $1^{\mathrm{f} / \mathrm{fl}}$ mice appear healthy and exhibit normal hematopoiesis for at least 12 weeks post-pIpC treatment [13]. This difference in overall survival between two studies may potentially be attributed to discrepancies in $\mathrm{pIpC}$ dosing regimen.

Based on competitive bone marrow transplant experiments, total engraftment potential of Dnmt1-KO HSCs towards peripheral blood contribution was significantly impaired over the period of 20 weeks post-transplant [13]. The lack of Dnmt1 in HSCs leads to a greater reduction in myeloid lineages as opposed to lymphoid lineages. It has been proposed that acute loss of the mature myeloid lineages caused by increased cycling coupled with defects in differentiation of Dnmt1-KO HSCs to replace these cells may be the major mechanism by which the myeloid pool is lost acutely [13]. In contrast with complete loss of Dnmt 1 in HSCs, mice with hypomorphic expression of Dnmt1 are viable and exhibit a milder impairment of hematopoiesis [12]. These data reveal that a critical threshold of DNA methylation is required to maintain HSC self-renewal, differentiation and proper lineage distribution.

\section{DNMT3A}

Initial retroviral-based studies suggested that conditional deletion of Dnmt3a or Dnmt3b in adult HSCs had no major impact on self-renewal and differentiation [14]. However, given the high prevalence of DNMT3A mutations in hematopoietic disorders and malignancies, the roles of both Dnmt $3 \mathrm{a} / 3 \mathrm{~b}$ in HSCs were more recently re-evaluated [15, 16]. Conditional deletion of Dnmt3a (henceforth referred to as Dnmt3a-KO) in hematopoiesis using the Mx1-Cre driver, expands adult HSC numbers upon serial transplantation while impairing their ability to differentiate on a per HSC basis. Although the ability of Dnmt3a-KO HSCs to differentiate is relatively impaired, their self-renewal potential is drastically increased with an average of tenfold enhancement after secondary transplantation that is persistent even after four rounds of transplantation. This increased selfrenewal potential of Dnmt3a-KO HSCs allows them to significantly outcompete their wild-type counterparts and accumulate in the bone marrow. In addition, most Dnmt3aKO HSCs in bone marrow are quiescent similar to wildtype HSCs, leading to notion that their competitive advantage arises from defects in cell fate decisions that control self-renewal vs differentiation [15]. Analysis of DNA methylation together with gene expression changes in Dnmt3aKO HSCs and their progeny suggested that the stem cellspecific genes were unable to be repressed in the absence of Dnmt3a, allowing the HSC self-renewal to be sustained and thereby inhibiting differentiation [15]. Some of the genes involved in HSC self-renewal, which might partly account 
for this phenotype, include Runx1, Gata3 and Nr4a21 [5]. It has been previously shown that overexpression of Runx 1 [17] and Gata3 [18] in normal HSCs inhibits differentiation, whereas $\mathrm{Nr} 4 \mathrm{a} 2$ overexpression forces HSCs into quiescence [19]. Although this work underlines the importance of Dnmt3a in HSC function, there are still several enigmatic aspects which remain unclear. For example, loss of Dnmt3a induced both hypo- and hyper-methylation within different promoters and there was little correlation between changes in methylation and differential gene expression in the mutant HSCs. It has been hypothesized that increase in methylation might be due to abnormal activity of Dnmt3b, suggesting that both de novo methyltransferases might function in synergy to regulate hematopoiesis [16].

\section{DNMT3B}

The function of Dnmt3b in hematopoiesis and its synergistic role with Dnmt3a is made available by the conditional inactivation of Dnmt3b (henceforth referred to as Dnmt3b-KO) in adult HSCs either alone or combination with Dnmt3a [16]. Loss of both Dnmt3a and Dnmt3b (henceforth referred to as dKO) in HSCs suggested a cooperative mode of action, which resulted in enhanced HSC self-renewal and a more severe block in differentiation than in Dnmt3a-KO HSCs, although loss of Dnmt3b alone did not produce a phenotype compared to control HSCs. When Dnmt3b-KO and dKO HSCs are forced differentiated in a transplant experiment, Dnmt3b-KO HSCs performed similarly to the control group in establishing peripheral blood chimerism, whereas dKO HSCs exhibited marked reduction in all lineages. The same transplant setting also revealed that dKO HSCs underwent extensive self-renewal, approximately fivefold higher in HSC numbers compared to Dnmt3a-KO HSCs on a per-cell basis. These phenotypes were distinct compared to Dnmt3a-KO HSCs, and these data reinforce the notion that Dnmt3a and Dnmt3b functionally cooperate in hematopoiesis to enable HSC differentiation. Transcriptome analysis of dKO HSCs suggested upregulation of the $\beta$-catenin signaling pathway via twofold upregulation of Ctnnb1, and increased expression of its target genes including Ccnd1, Ppar6, Vegf $\alpha$ and Jag1 [16]. Constitutive activation of the $\beta$-catenin signaling pathway has been previously implicated in HSC function [20], and has similar phenotypes to dKO HSCs, such as up to a 15-fold increase in HSC numbers and a sharp differentiation block [20]. These data suggest that activated $\beta$-catenin signaling may be partly responsible for the differentiation deficit of dKO HSCs. Indeed, downregulation of Ctnnbl in dKO HSCs can partly restore the differentiation potential of $\mathrm{dKO}$ in vitro, suggesting that the $\beta$-catenin pathway contributes to HSC fate determination through potential epigenetic regulation via DNA methylation.
Based on these findings, it can be concluded that hematopoietic homeostasis is at least partly regulated by DNA methylation-dependent control mechanisms, and all DNMTs are required for HSCs to undergo proper functional development. For the sake of simplicity, we also summarized effect of most KO animal models available for DNMTs to date on normal and malignant hematopoiesis (Table 1).

\section{Role of DNMTs in malignant hematopoiesis}

\section{DNMT3A}

Since the early reports of DNMT3A mutations in AML and MDS patients in 2010 [21-23], mutations in DNMT3A have been frequently reported across a range of hematopoietic diseases. Somatic mutations in DNMT3A occur in approximately $22 \%[21,24], 10 \%[25,26]$, and $8 \%$ [27, 28], of patients with AML, MDS, and MPN, respectively, and are associated with poor prognosis. Although a variety of mutations including missense, frame-shift, nonsense, and splice-site mutations were identified throughout the gene [29], the most prevalent mutation (or a mutational hotspot) involved a substitution at residue 882 in the methyltransferase domain, generating the $\mathrm{R} 882 \mathrm{H}$ variant (Fig. 1). This mutation acts as a dominant negative resulting in reduced catalytic activity [30, 31]. The functional relevance of most alternative mutations across this gene on promoting leukemogenesis are yet to be explored. Although it is widely accepted that DNMT3A loss in hematopoiesis is considered detrimental, there is still no consensus on the exact molecular pathways involved with reduced DNMT3A activity in AML patients. A correlation has not yet been found between DNA methylation and gene expression in DNMT3A-mutant AML cells, which contributes to pathogenesis. A number of studies reported that there is no clearly defined cohort of genes or microRNA expression signatures which are aberrantly regulated in between AML patients with DNMT3A mutations [32, 33]. Due to the aforementioned lack of clarity in the role of DNMT3A mutations in AML, it has been challenging thus far to design and develop rationale for targeted therapies for DNMT3A-mutant AML patients. However, with the increasing availability of novel mouse models, researchers may soon be able to overcome these conflicts and gain a better understanding of role of DNMT3A mutations in hematological malignancy in the near future.

\section{DNMT3A mutations in clonal hematopoiesis of indeterminate potential (CHIP)}

Although molecular programs are tightly regulated during hematopoietic homeostasis, cells exhibiting the CHIP 


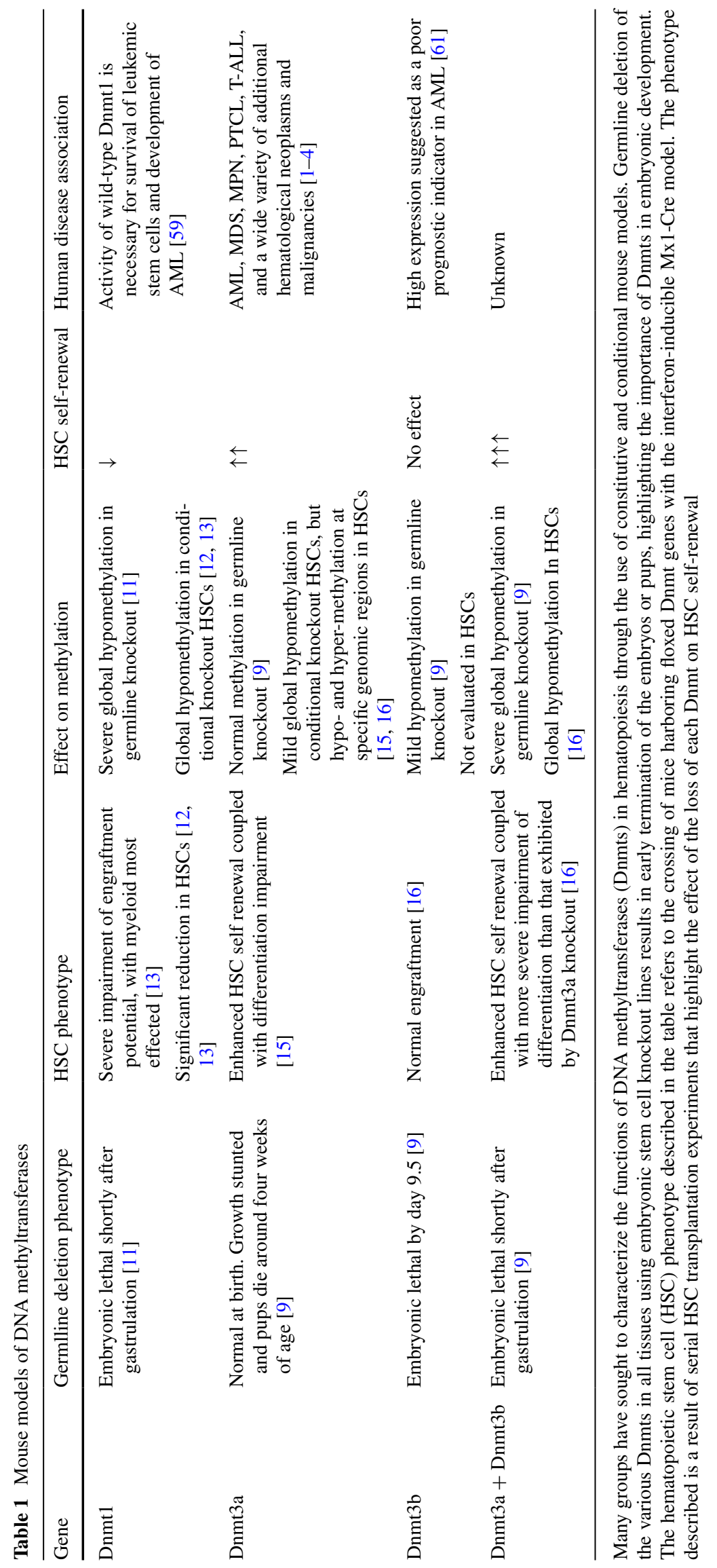




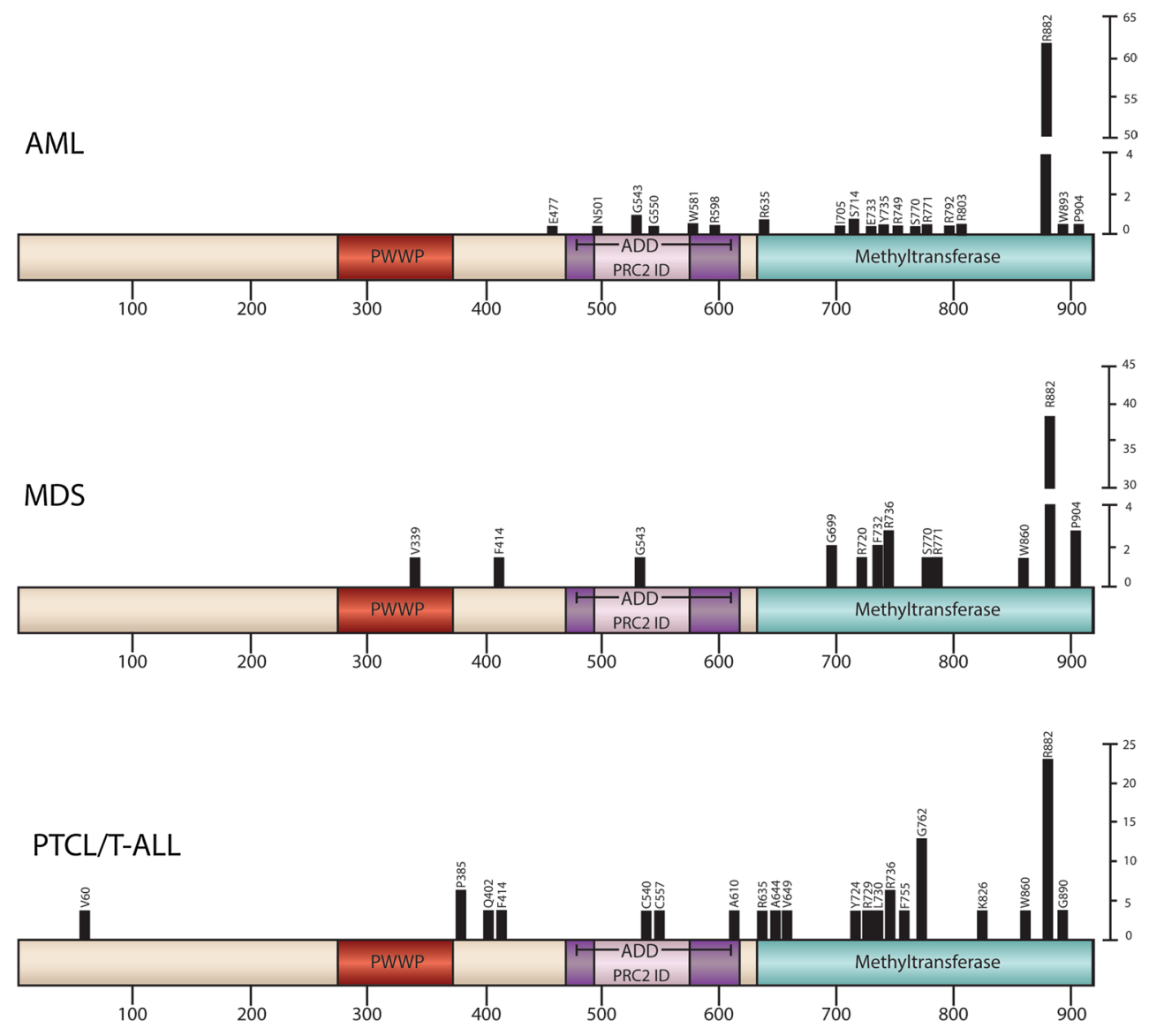

Fig. 1 Distribution of somatic mutations in DNMT3A in hematological malignancies. Schematic representation of DNMT3A primary structure with values along the bottom representing amino acid residues. Highlighted along the top are mutation frequencies from the Catalogue of Somatic Mutations in Cancer (COSMIC) for AML, MDS, and combined PTCL/T-ALL as T cell neoplasms. Scale to the right indicates percent of patients out of the data set with a given mutation. The AML data set represents the 19 most frequently mutated residues in a cohort of 1532 AML patients with mutations in DNMT3A (selected based on occurrence of five or more mutation

phenotype arise from hematopoietic and stem progenitor cells (HSPCs) due to genetic and epigenetic alterations, giving rise to a pool of malignant cells that are characterized by uncontrolled proliferation and/or abnormal differentiation (Fig. 2). Sequencing studies of hematological malignancies indicated that DNMT3A mutations are typically found at higher variant allele frequencies than other accompanying mutations $[34,35]$. This ratio of allelic variance suggests that DNMT3A mutations arise early in leukemic evolution of HSPCs, potentially in HSCs, leading to a pool of dominant clones from which AML evolves. A number of xenograft transplantation studies suggested that HSCs with DNMT3A mutations obtained from AML patients exhibit a marked advantage compared to wild-type instances at a given residue). The MDS data set represents all mutations occurring with a frequency of two or more in a data set of 143 MDS patients with mutations in DNMT3A. The PTCL/T-ALL data set represents 14 and 17 patients with PTCL and T-ALL, respectively, which included 20 distinct DNMT3A mutations. Frequencies of all 20 different DNMT3A mutations were calculated. The majority of mutations in all diseases are clustered into two domains along the gene, the ADD and the methyltransferase domains. The most frequent mutation found in all data sets represented for patients with DNMT3A mutations falls within this domain at residue R882

HSCs [35, 36], similar to Dnmt3a-KO murine models [15]. In the absence of common leukemia-associated mutations, these clones can still contribute to multiple blood lineages, while existing in a pre-leukemic state prior to acquisition of secondary co-operating mutations that induce leukemia [36]. Over the past few years, in vivo KO animal models greatly improved our understanding of the role of DNMT3A mutations in hematopoietic malignancies. In this section, we will summarize recent studies supporting the notion that HSCs which lack Dnmt3a can exist in a state of pre-leukemic potential. These clones can further acquire leukemia-associated mutations in other genes which can cooperate with DNMT3A mutations to drive multiple types of hematological malignancies $[37,38]$. 


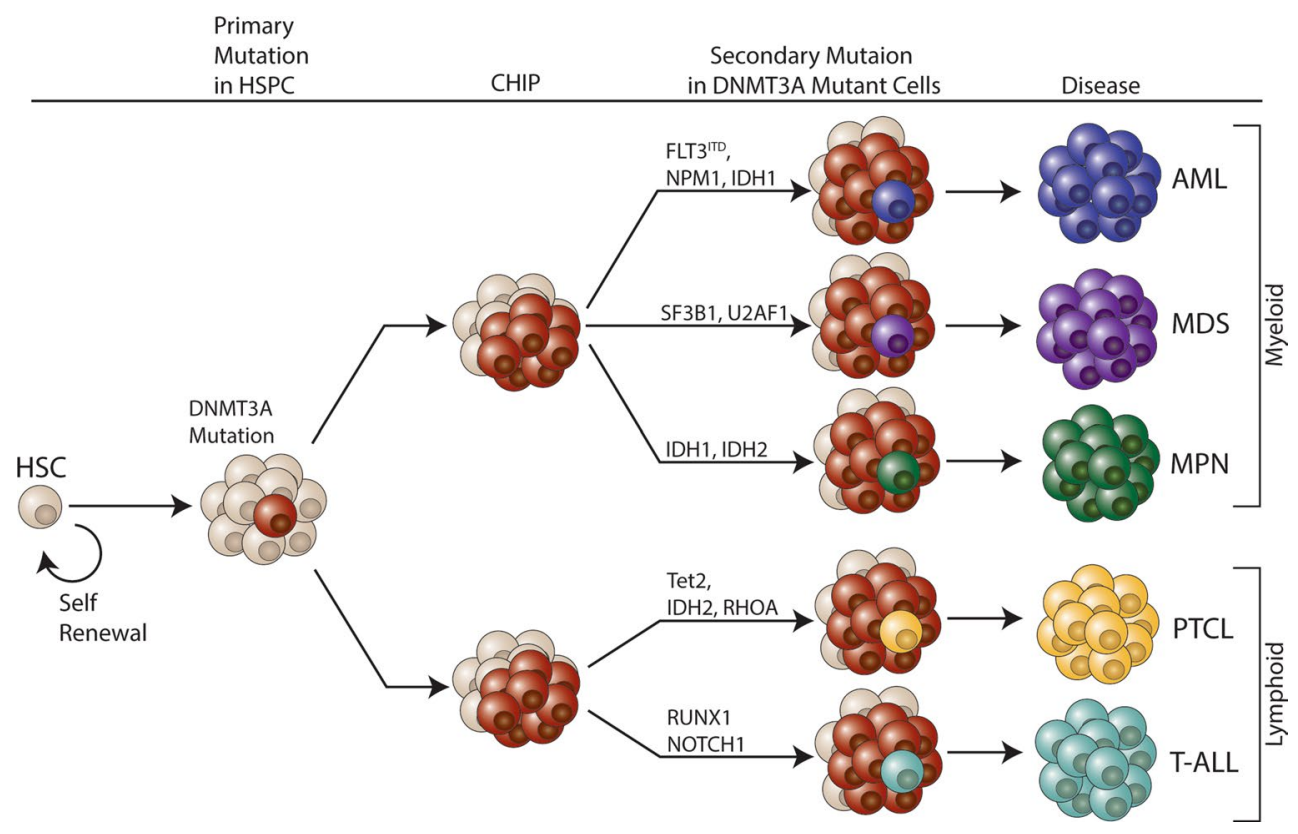

Fig. 2 Mutations in DNMT3A may cooperate with secondary mutations to promote variety of hematopoietic diseases. Mutations in DNMT3A are thought to be acquired at the level of hematopoietic stem or progenitor cells (HSPCs), possibly in HSCs, giving rise to pool of dominant clones leading to clonal hematopoiesis of indeterminate potential (CHIP). In the absence of common leukemia-asso-

\section{Co-operating mutational patterns of DNMT3A}

The mutational profiling of large AML patient cohorts has revealed that the most frequently co-mutated genes in DNMT3A-mutant AML are NPMI, FLT3 and IDHI. Approximately, $60 \%$ of AML patients with DNMT3A mutations also bear mutations in the NPM1 gene, whereas only $13 \%$ of non-DNMT3A-mutant AML patients carry NPM1 mutations [21, 24, 39]. These data suggest that co-operating mutations that associate with DNMT3A mutations occur in a non-random and selective fashion. In contrast, despite their high prevalence in AML, DNMT3A mutations are almost never found or negatively correlated with mutations that involve MLL, ASXL1 [40$42]$, or the chromosomal translocations involving $t(15 ; 17)$, $\operatorname{inv}(16)$ and $t(8 ; 21)$ [21, 23, 24, 43]. In addition to AML, DNMT3A mutations are also present in other hematological disorders such as MDS, MPN, T-ALL and peripheral T cell lymphoma (PTCL) (Fig. 2). Mutations in DNMT3A can cooperate with secondary mutations in splicing factor genes such as SF3B1 and U2AF1, or IDH1/2 to drive MDS and MPN, respectively [32, 44-46]. Mutations in TET2, IDH2, and RHOA or in RUNX1 and NOTCH1 in combination with DNMT3A mutations are also often found in PTCL and T-ALL patients, respectively [47-50]. ciated mutations, these clones can still contribute to multiple blood lineages, while existing in a state of CHIP. Once additional novel mutations are acquired, these clones may give rise to number of different hematopoietic diseases. There are several co-operating mutational patterns that have tendencies to give rise to specific hematopoietic diseases as shown

Identification of mutations in human patient samples using genome sequencing studies does not establish whether genomic alterations are a cause of the leukemia due to complexity of mutational spectrum. The contribution of these alterations is often experimentally validated in murine models by studying their co-operativity in HSPCs with a Dnmt3a mutant genetic background. Two of the most recent studies suggested that irradiated mice transplanted with Dnmt3a-KO HSCs in the absence of whole BM support succumbed to BM failures and died within a year [37, 38]. These animals were diagnosed with a spectrum of hematological diseases similar to those seen in patients harboring DNMT3A mutations, including MDS, MPN, AML and $\mathrm{T}$ cell acute lymphocytic leukemia (T-ALL). Some animals acquired secondary mutations which likely determined the lineage of the malignancy including co-operating mutations similar to those identified in patients including Npm1, Nras, Notch1 and c-Kit. In these studies, co-operativity of some of these mutations were experimentally validated using ectopic expression of $\mathrm{Nras}^{\mathrm{G} 12 \mathrm{D}}$ in Dnmt3a-KO background, which led to the development of AML-like phenotype [38], whereas c-Kit ${ }^{\mathrm{V} 750 \mathrm{M}}$ expression in Dnmt3anull HSPCs produced leukemias of various lineages [37]. The use of these mouse models will allow us to understand genetic processes associated with the progression of 
AML and may help guide research avenues for targeted therapeutics.

\section{Prognostic potential of DNMT3A mutations}

Numerous studies have been carried out to determine whether DNMT3A mutations hold prognostic potential in a clinically relevant context. Most of these studies looked at age, overall survival (OS), impact on complete remission (CR) rate, and relapse-free survival (RFS) status between the patients with and without the DNMT3A mutations $[21,32,33,39,51,52]$. However, it is challenging to compare these studies due to large variations between patient populations, treatment regiments and type of the methods used to investigate. Most of these studies agree that there is no statistically significant difference between CR, RFS between the patients with or without the DNMT3A mutations. However, most studies found that the median OS of DNMT3A-mutant AML patients is significantly lower compared to those with wild-type DNMT3A regardless of age (12.3 vs. 41.1 months with a $p$ value of $<0.001)[32,33,39$, $52]$, even though OS rate varies depending on the study due to varying cohort size. These data clearly suggest that presence of DNMT3A mutations is an independent unfavorable prognostic factor for OS. In addition, the most commonly mutated amino acid residue in DNMT3A-mutant AML, R882, appears to be inconclusive in terms of the prognostic impact relative to patients with non-R882 DNMT3A mutations $[33,53]$. Despite the lack of consensus between each study, some found that there may be some prognostic value when DNMT3A-mutant AML is analyzed in the presence and absence of the most commonly mutated co-operating events. Several groups have shown a strong positive correlation between occurrence of DNMT3A mutations and mutations in NPM1 or FLT3, most commonly the FLT3 ${ }^{\text {ITD }}$ variant which harbors internal tandem duplication (ITD) of the juxtamembrane domain leading to constitutive activation [21, 43]. Interestingly, Ribiero and colleagues have shown that patients with DNMT3A-mutant AML in combination with wild-type NPM1 and/or wild-type FLT3 have a significant inferior OS and RFS [39]. In contrast, patients with DNMT3A-mutant AML together with mutant NPM1 and wild-type FLT3 or with mutant NPM1 and FLT3 ${ }^{\text {ITD }}$ have no prognostic value. This is one of the largest comprehensive studies to date indicating prognostic implications of these combinations of mutations [39].

\section{Therapeutic implications}

Therapeutic resistance of DNMT3A-mutant AML to standard first-line chemotherapy remains largely enigmatic due to their lack of efficacy. Recent studies have indicated that wild-type DNMT3A acts as a pro-apoptotic switch in response to genotoxic stress $[54,55]$, giving rise to hypothesis that loss of DNMT3A may protect cells from apoptosis in response to DNA damage caused by possible alterations in mechanisms such as DNA-damage sensing, response and repair. For most AML treatment regiments, DNA-damaging anthracyclines appear to be an effective line of therapy. Studies suggest that DNMT3A-mutant AML patients have better therapeutic outcome when they undergo high-dose DNA-damaging anthracycline therapy compared to standard dose regiments [24, 56]. However, use of high-dose DNA-damaging regiments comes at the cost of increased somatic toxicity and do not completely cure the disease.

Other therapeutic agents, such as DNA methyltransferase inhibitors (5-azacytidine and its derivative decitabine) have been in use for the treatment of MDS and recently being tested for the treatment of AML [33, 57, 58]. These inhibitors are thought to work by inhibiting the function of DNMT1, and ultimately inducing hypomethylation of leukemia-associated genes, which are aberrantly silenced in disease. Although studies with a large number of patients with DNMT3A mutations are necessary to define exact therapeutic outcomes of these inhibitors, a number of small-scale studies indicated that treatment of DNMT3A-mutant AML patients with decitabine resulted in a higher RFS and a slight increase in OS [33].

AML is complex and heterogeneous disease with a number of potential genetic factors being involved in its progression. Regardless of the treatment regimens available to DNMT3A-mutant AML patients, there is no clear impact of these treatments to achieve complete remission. Rather than using a "one size fits all" treatment approach for these patients, improved mechanistic understanding of the mutations present in DNMT3A-mutant AML may lead to advances of therapeutics in the field of personalized cancer treatments.

\section{DNMT1}

It was not clear until recently with the use of an MLLAF9-induced AML mouse model that some DNA methylation patterns unique to leukemia stem cells (LSCs) required for their survival are established by the maintenance methyltransferase DNMT1 [59]. Trowbridge and colleagues have demonstrated that the ablation of Dnmt1 in the MLL-AF9 model completely hindered AML transformation while the control group with wild-type Dnmt1 developed fully penetrant AML with a median survival of 81 days [59]. These data clearly suggested that MLLAF9 LSC survival is dependent on methylation patterns established by Dnmt1. To further evaluate the effect of Dnmt1 ablation on the maintenance of pre-existing AML, Trowbridge and colleagues transplanted leukemic granulocyte-macrophage progenitors (L-GMPs) into recipients 
to establish AML. Once established, they conditionally deleted Dnmt1 in recipients. Data indicated that loss of Dnmt1 in L-GMPs induced a significant delay in the progression of pre-existing AML, while doubling the survival time compared to control cohorts. Moreover, when AML cells that lacked Dnmt1 were transplanted into secondary recipients, their ability to re-establish the disease was also significantly hindered with a survival time extended by twofold. The authors hypothesized that Dnmtl haploinsufficiency or pharmacologic inhibition of Dnmt1 activity can be made available in a clinically relevant context and in fact, ablation of one Dnmt1 allele or pharmacologic inhibition of Dnmt1 activity using decitabine and zebularine (DNA methylation inhibitors) provided similar data impairing LSC maintenance and function. To further elucidate the molecular programs associated with the LSC dependence on Dnmt1, authors performed genome-wide expression profiling approach, which revealed that Dnmtl haploinsufficiency induced derepression of multiple genes in MLL-AF9 LSCs including Dusp6, Klf4, Tgfbi, and Timp2, all of which are cancer-related tumor suppressors and their downregulation is likely to contribute LSC survival [59]. Peters and colleagues also provided evidence suggesting that Dnmt1-mediated DNA methylation is crucial for the survival for lymphoid malignancies. Using a mouse model of MYC-induced $\mathrm{T}$ cell lymphomagenesis, ablation of Dnmt1 in primary lymphoma cells impaired tumor cell proliferation by inducing rapid apoptosis in vivo [60].

Together, these data indicate that Dnmt1-mediated DNA methylation provides epigenetic control of genes involved in leukemia self-renewal and progression and targeting DNMT1 could provide therapeutic index for patients with lymphoid and myeloid malignancies. However, the dependency of leukemias with different genetic backgrounds remains to be defined.

\section{DNMT3B}

While specific mutations in DNMT3B have not yet been indicated as a causal factor in hematological malignancies, aberrant expression of DNMT3B and its various isoforms has been associated with poor prognosis in AML and the acceleration of lymphomagenesis in $\mathrm{T}$ and $\mathrm{B}$ cell lymphomas. It has recently been reported that high DNMT3B expression is significantly correlated with poor prognostic outcomes in cytogenetically normal AML in older patients [61]. Although high expression of DNMT3B is linked with poor prognosis in AML, caution is required in interpreting these data due to the existence wide array of catalytically inactive DNMT3B isoforms that have been reported to have pathogenic qualities [62]. DNMT3B4 and 3B2 are isoforms of DNMT3B belonging to a group of DNMT3B isoforms, termed DNMT3Bs. Expression of these two DNMT3Bs was shown to be strongly correlated with tumor suppressor promoter methylation in non-small cell lung carcinoma. $91 \%$ of tumors containing methylated RASSF1A promoters expressed DNMT3B4 and the remaining $9 \%$ not expressing DNMT3B4, expressed DNMT3B2 [62]. There has been no report of DNMT3B expression in hematological malignancies to date, but this family of DNMT3Bs may provide insight into differentiation defects and clonal expansion in leukemias and lymphomas. One truncated DNMT3B isoform, DNMT3B7, has been indicated as a potential dominant negative, leading to an accelerated rate of lymphomagenesis in E $\mu-\mathrm{Myc}$ mice [63]. This same study also suggested that DNMT3B itself acts as a haploinsufficient tumor suppressor, in that DNMT3B \pm mice had an increased rate of lymphomagenesis [63]. The role of DNMT3B as an indirect tumor suppressor was also indicated in a mouse model of MYC-induced T cell lymphoma by its function in methylation of the lymphoma proliferation gene Ment. Complete loss of DNMT3B caused a stepwise demethylation in the gene Ment and an acceleration of lymphomagenesis [64]. As indicated in the aforementioned studies, the role of DNMT3B and its isoforms in hematologic malignancies has yet to be definitively elucidated. While some isoforms appear to have oncogenic properties due to tumor suppressor promoter methylation, others indicate DNMT3B as having indirect roles as a tumor suppressor itself. Due to the contradictory results and ambiguity of the regulation of DNMT3B and its isoforms, further investigation is needed to clarify its role in the context of hematological malignancies.

\section{Concluding remarks}

Epigenetic regulation in hematopoiesis has been a field of rapid expansion in recent decades, aided by the advances in sequencing technologies and development of novel mouse models. Literature reviewed here demonstrates our current knowledge of the overlapping and distinct roles that the DNA methyltransferase enzymes DNMT1, DNMT3A, and DNMT3B play in normal and malignant hematopoiesis.

While the maintenance methyltransferase DNMT1 and the de novo methyltransferase DNMT3B have been shown to exhibit important roles in normal hematopoiesis, there still remains much to be elucidated about their functions in hematological malignancies. In contrast, mutations in DNMT3A are frequent events in hematological diseases and mouse models have indicated that DNMT3A controls the balance between HSC self-renewal vs differentiation in normal hematopoiesis. When this balance is disrupted, DNMT3A-mutant hematopoietic stem and progenitor cells are potentially predisposed to development of hematological diseases, including AML. What remains is to gain 
improved mechanistic understanding of how co-operating mutations collaborate with DNMT3A to cause the spectrum of diseases. This will allow scientists to design and develop effective treatments for patients, rather than using "one size fits all" treatment approaches, which are ineffective and comes at the cost of increased toxicity. Future research will seek to fill these gaps in knowledge and surely improve our understanding of epigenetics in hematopoiesis.

\section{References}

1. Yang L, Rau R, Goodell MA. DNMT3A in haematological malignancies. Nat Rev Cancer. 2015;15:152-65.

2. Beerman I, Rossi DJ. Epigenetic regulation of hematopoietic stem cell aging. Exp Cell Res. 2014;329:192-9.

3. Sashida G, Iwama A. Epigenetic regulation of hematopoiesis. Int J Hematol. 2012;96:405-12.

4. Cullen SM, Mayle A, Rossi L, Goodell MA. Hematopoietic stem cell development: an epigenetic journey. Curr Top Dev Biol. 2014;107:39-75.

5. Jaenisch R. DNA methylation and imprinting: why bother? Trends Genet. 1997;13:323-9.

6. Schaefer CB, Ooi SK, Bestor TH, Bourc'his D. Epigenetic decisions in mammalian germ cells. Science. 2007;316:398-9.

7. Okano M, Xie S, Li E. Cloning and characterization of a family of novel mammalian DNA (cytosine-5) methyltransferases. Nat Genet. 1998;19:219-20.

8. Hata K, Okano M, Lei H, Li E. Dnmt3L cooperates with the Dnmt3 family of de novo DNA methyltransferases to establish maternal imprints in mice. Development. 2002;129:1983-93.

9. Okano M, Bell DW, Haber DA, Li E. DNA methyltransferases Dnmt3a and Dnmt3b are essential for de novo methylation and mammalian development. Cell. 1999;99:247-57.

10. Bestor TH. Activation of mammalian DNA methyltransferase by cleavage of a $\mathrm{Zn}$ binding regulatory domain. EMBO J. 1992;11:2611-7.

11. Li E, Bestor TH, Jaenisch R. Targeted mutation of the DNA methyltransferase gene results in embryonic lethality. Cell. 1992;69:915-26.

12. Broske AM, et al. DNA methylation protects hematopoietic stem cell multipotency from myeloerythroid restriction. Nat Genet. 2009;41:1207-15.

13. Trowbridge JJ, Snow JW, Kim J, Orkin SH. DNA methyltransferase 1 is essential for and uniquely regulates hematopoietic stem and progenitor cells. Cell Stem Cell. 2009;5:442-9.

14. Tadokoro Y, Ema H, Okano M, Li E, Nakauchi H. De novo DNA methyltransferase is essential for self-renewal, but not for differentiation, in hematopoietic stem cells. J Exp Med. 2007;204:715-22.

15. Challen GA, et al. Dnmt3a is essential for hematopoietic stem cell differentiation. Nat Genet. 2012;44:23-31.

16. Challen GA, et al. Dnmt3a and Dnmt3b have overlapping and distinct functions in hematopoietic stem cells. Cell Stem Cell. 2014;15:350-64.

17. Challen GA, Goodell MA. Runx1 isoforms show differential expression patterns during hematopoietic development but have similar functional effects in adult hematopoietic stem cells. Exp Hematol. 2010;38:403-16.

18. Chen D, Zhang G. Enforced expression of the GATA-3 transcription factor affects cell fate decisions in hematopoiesis. Exp Hematol. 2001;29:971-80.
19. Sirin O, Lukov GL, Mao R, Conneely OM, Goodell MA. The orphan nuclear receptor Nurr1 restricts the proliferation of haematopoietic stem cells. Nat Cell Biol. 2010;12:1213-9.

20. Scheller M, et al. Hematopoietic stem cell and multilineage defects generated by constitutive beta-catenin activation. Nat Immunol. 2006;7:1037-47.

21. Ley TJ, et al. DNMT3A mutations in acute myeloid leukemia. $\mathbf{N}$ Engl J Med. 2010;363:2424-33.

22. Yamashita $Y$, et al. Array-based genomic resequencing of human leukemia. Oncogene. 2010;29:3723-31.

23. Yan XJ, et al. Exome sequencing identifies somatic mutations of DNA methyltransferase gene DNMT3A in acute monocytic leukemia. Nat Genet. 2011;43:309-15.

24. Patel JP, et al. Prognostic relevance of integrated genetic profiling in acute myeloid leukemia. N Engl J Med. 2012;366:1079-89.

25. Walter MJ, et al. Recurrent DNMT3A mutations in patients with myelodysplastic syndromes. Leukemia. 2011;25:1153-8.

26. Ewalt M, et al. DNMT3a mutations in high-risk myelodysplastic syndrome parallel those found in acute myeloid leukemia. Blood Cancer J. 2011;1:e9.

27. Abdel-Wahab O, et al. DNMT3A mutational analysis in primary myelofibrosis, chronic myelomonocytic leukemia and advanced phases of myeloproliferative neoplasms. Leukemia. 2011;25:1219-20.

28. Stegelmann F, et al. DNMT3A mutations in myeloproliferative neoplasms. Leukemia. 2011;25:1217-9.

29. Forbes SA, et al. COSMIC: exploring the world's knowledge of somatic mutations in human cancer. Nucleic Acids Res. 2015;43:D805-11.

30. Kim SJ, et al. A DNMT3A mutation common in AML exhibits dominant-negative effects in murine ES cells. Blood. 2013;122:4086-9.

31. Russler-Germain DA, et al. The R882H DNMT3A mutation associated with AML dominantly inhibits wild-type DNMT3A by blocking its ability to form active tetramers. Cancer Cell. 2014;25:442-54.

32. Thol $\mathrm{F}$, et al. Incidence and prognostic influence of DNMT3A mutations in acute myeloid leukemia. $\mathrm{J}$ Clin Oncol. 2011;29:2889-96

33. Marcucci G, et al. Age-related prognostic impact of different types of DNMT3A mutations in adults with primary cytogenetically normal acute myeloid leukemia. J Clin Oncol. 2012;30:742-50.

34. Ding L, et al. Clonal evolution in relapsed acute myeloid leukaemia revealed by whole-genome sequencing. Nature. 2012;481:506-10.

35. Welch JS, et al. The origin and evolution of mutations in acute myeloid leukemia. Cell. 2012;150:264-78.

36. Shlush LI, et al. Identification of pre-leukaemic haematopoietic stem cells in acute leukaemia. Nature. 2014;506:328-33.

37. Celik H, et al. Enforced differentiation of Dnmt3a-null bone marrow leads to failure with c-Kit mutations driving leukemic transformation. Blood. 2015;125:619-28.

38. Mayle A, et al. Dnmt3a loss predisposes murine hematopoietic stem cells to malignant transformation. Blood. 2015;125:629-38.

39. Ribeiro AF, et al. Mutant DNMT3A: a marker of poor prognosis in acute myeloid leukemia. Blood. 2012;119:5824-31.

40. Bejar R, et al. Validation of a prognostic model and the impact of mutations in patients with lower-risk myelodysplastic syndromes. J Clin Oncol. 2012;30:3376-82.

41. Haferlach T, et al. Landscape of genetic lesions in 944 patients with myelodysplastic syndromes. Leukemia. 2014;28:241-7.

42. Damm F, et al. Mutations affecting mRNA splicing define distinct clinical phenotypes and correlate with patient outcome in myelodysplastic syndromes. Blood. 2012;119:3211-8. 
43. Gaidzik VI, et al. Clinical impact of DNMT3A mutations in younger adult patients with acute myeloid leukemia: results of the AML Study Group (AMLSG). Blood. 2013;121:4769-77.

44. Thol F, et al. Frequency and prognostic impact of mutations in SRSF2, U2AF1, and ZRSR2 in patients with myelodysplastic syndromes. Blood. 2012;119:3578-84.

45. Fried I, et al. Frequency, onset and clinical impact of somatic DNMT3A mutations in therapy-related and secondary acute myeloid leukemia. Haematologica. 2012;97:246-50.

46. Zebisch A, Hoefler G, Quehenberger F, Wolfler A, Sill H. Mutant DNMT3A in acute myeloid leukemia: guilty of inducing genetic instability? Leukemia. 2013;27:1777-8.

47. Odejide $\mathrm{O}$, et al. A targeted mutational landscape of angioimmunoblastic T-cell lymphoma. Blood. 2014;123:1293-6.

48. Palomero $\mathrm{T}$, et al. Recurrent mutations in epigenetic regulators, RHOA and FYN kinase in peripheral $\mathrm{T}$ cell lymphomas. Nat Genet. 2014;46:166-70.

49. Sakata-Yanagimoto M, et al. Somatic RHOA mutation in angioimmunoblastic T cell lymphoma. Nat Genet. 2014;46:171-5.

50. Grossmann V, et al. The molecular profile of adult T-cell acute lymphoblastic leukemia: mutations in RUNX1 and DNMT3A are associated with poor prognosis in T-ALL. Genes Chromosomes Cancer. 2013;52:410-22.

51. Shen Y, et al. Gene mutation patterns and their prognostic impact in a cohort of 1185 patients with acute myeloid leukemia. Blood. 2011;118:5593-603.

52. Hou HA, et al. Integration of cytogenetic and molecular alterations in risk stratification of 318 patients with de novo non-M3 acute myeloid leukemia. Leukemia. 2014;28:50-8.

53. Renneville A, et al. Prognostic significance of DNA methyltransferase $3 \mathrm{~A}$ mutations in cytogenetically normal acute myeloid leukemia: a study by the Acute Leukemia French Association. Leukemia. 2012;26:1247-54.
54. Zhang Y, et al. DNMT3a plays a role in switches between doxorubicin-induced senescence and apoptosis of colorectal cancer cells. Int J Cancer. 2011;128:551-61.

55. Jin B, Robertson KD. DNA methyltransferases, DNA damage repair, and cancer. Adv Exp Med Biol. 2013;754:3-29.

56. LaRochelle O, et al. Do AML patients with DNMT3A exon 23 mutations benefit from idarubicin as compared to daunorubicin? A single center experience. Oncotarget. 2011;2:850-61.

57. Traina $\mathrm{F}$, et al. Impact of molecular mutations on treatment response to DNMT inhibitors in myelodysplasia and related neoplasms. Leukemia. 2014;28:78-87.

58. Klco JM, et al. Genomic impact of transient low-dose decitabine treatment on primary AML cells. Blood. 2013;121:1633-43.

59. Trowbridge JJ, et al. Haploinsufficiency of Dnmt1 impairs leukemia stem cell function through derepression of bivalent chromatin domains. Genes Dev. 2012;26:344-9.

60. Peters SL, et al. Essential role for Dnmt1 in the prevention and maintenance of MYC-induced T-cell lymphomas. Mol Cell Biol. 2013;33:4321-33.

61. Niederwieser $\mathrm{C}$, et al. Prognostic and biologic significance of DNMT3B expression in older patients with cytogenetically normal primary acute myeloid leukemia. Leukemia. 2015;29:567-75.

62. Wang J, Walsh G, Liu DD, Lee JJ, Mao L. Expression of Delta DNMT3B variants and its association with promoter methylation of p16 and RASSF1A in primary non-small cell lung cancer. Cancer Res. 2006;66:8361-6.

63. Vasanthakumar A, et al. Dnmt3b is a haploinsufficient tumor suppressor gene in Myc-induced lymphomagenesis. Blood. 2013;121:2059-63.

64. Hlady RA, et al. Loss of Dnmt3b function upregulates the tumor modifier Ment and accelerates mouse lymphomagenesis. J Clin Invest. 2012;122:163-77. 